\title{
Rhabdomyolysis: Patterns, Circumstances, and Outcomes of Patients Presenting to the Emergency Department
}

\author{
Emily G. Knafl, BA, ${ }^{1}$ James A. Hughes, RN, BN, ${ }^{2,3}$ Goce Dimeski, PhD, FFSc, (RCPA), ${ }^{4,5}$ \\ Rob Eley, BSc, MSc, PhD, CSci, CBiol, FRSB ${ }^{5,6}$ \\ ${ }^{1}$ The University of Queensland, Ochsner Clinical School, Brisbane, Queensland, Australia and New Orleans, LA ${ }^{2}$ Emergency and Trauma \\ Centre, Royal Brisbane and Women's Hospital, Herston, Queensland, Australia ${ }^{3}$ Queensland University of Technology, Kelvin Grove Campus \\ - School of Nursing, Kelvin Grove, Queensland, Australia ${ }^{4}$ Department of Chemical Pathology, Princess Alexandra Hospital, Woolloongabba, \\ Queensland, Australia ${ }^{5}$ The University of Queensland, Faculty of Medicine, Herston, Brisbane, Queensland, Australia ${ }^{6}$ Emergency \\ Department, Princess Alexandra Hospital, Woolloongabba, Queensland, Australia
}

Background: Exertional rhabdomyolysis is a condition resulting from skeletal muscle damage and release of intracellular toxins into the systemic circulation as a consequence of extreme physical effort. With increasing numbers of people partaking in highintensity workouts, we hypothesized that the rate of presentation of exertional rhabdomyolysis was also increasing.

Methods: All presentations to the Princess Alexandra Hospital emergency department in Brisbane, Australia between March 2005 and December 2016 were identified from the electronic medical record. Records of patients with a serum creatine kinase value $>1,000 \mathrm{U} / \mathrm{L}$ or a urinary myoglobin $>5 \mathrm{mg} / \mathrm{L}$ were extracted for determination of whether the rhabdomyolysis was attributable to physical exertion.

Results: From a total of 1,957 rhabdomyolysis cases, 89 patients ( $70.8 \%$ male) were identified as having exertional rhabdomyolysis. The frequency of presentation increased from $0.28 / 10,000$ presentations in 2005 to 3.5/10,000 in 2015. Gym-induced exertional rhabdomyolysis was the primary subcategory (53.9\%) for these cases, and manual labor was the second most common subcategory (15.7\%).

Conclusion: This study provides evidence for increasing instances of exertional rhabdomyolysis in the Brisbane, Australia metropolitan area and adds to the current knowledge about those who develop exertional rhabdomyolysis. Future studies are warranted to investigate if similar trends are being seen in other regions and to identify the circumstances leading to the presentation. Such knowledge would be valuable for devising strategies to prevent and mitigate injury.

Keywords: Creatine kinase, exercise, myoglobin, rhabdomyolysis

Address correspondence to James A. Hughes, RN, BN, Nurse Research Manager, Emergency and Trauma Centre, Royal Brisbane and Women's Hospital, Butterfield Street, Herston, Queensland, Australia. Tel: +61-409-356-098. Email: james.hughes@health.qld.gov.au

\section{INTRODUCTION}

Exertional rhabdomyolysis, also frequently referred to as exercise-induced rhabdomyolysis, is a potentially lifethreatening condition resulting from skeletal muscle damage and the ensuing release of intracellular toxins into the systemic circulation as a consequence of extreme physical effort. ${ }^{1}$ The classic clinical triad of rhabdomyolysis includes myalgia, weakness, and pigmenturia. ${ }^{2}$ The mechanism attributed to these features includes muscle injury as a result of direct mechanical damage and indirect damage from energy depletion within the myocytes. ${ }^{1}$ Complications can range from mild discomfort to acute renal failure and severe electrolyte disturbances. ${ }^{3}$ Laboratory diagnostics involve either a urinary myoglobin $>5 \mathrm{mg} / \mathrm{L}$ or an elevated creatine kinase $(\mathrm{CK})$ value $>1,000 \mathrm{U} / \mathrm{L}$ (a 5-fold increase above normal physiologic levels). ${ }^{4}$ Early recognition allows for aggressive treatment of the underlying etiology and fluid replacement that can avert or mitigate renal damage and other complications. ${ }^{2}$

Exertional rhabdomyolysis has been well documented in the military and among professional and nonprofessional athletes. ${ }^{5,6}$ Various activities have been identified as the precipitating cause, including marathons, ${ }^{7}$ training by young athletes, ${ }^{8}$ stationary cycling, ${ }^{9}$ and extremely rigorous gymbased workouts. ${ }^{10}$

The majority of the literature on exertional rhabdomyolysis consists of case reports, with only 2 retrospective medical record studies from Norway investigating changes in incidences over time. The first study found that incidences of exertional rhabdomyolysis -identified based on CK laboratory values $>5,000 \mathrm{U} / \mathrm{L}$-increased 4-fold from 2011-2014. ${ }^{11}$ The second study, conducted between 2010 and 2015, also identified an 
upward trend in exertional rhabdomyolysis. ${ }^{12}$ In that study, 25 of the 33 patients who had a CK level 10 times the upper normal reference limit appeared in the last year of the study. This trend is also reflected in case reports that have increased in number and indicate the causal mechanism to be high-intensity training. ${ }^{12,13}$

Although causes of exertional rhabdomyolysis are well documented, case series and data describing changes over time are limited. To add to the current evidence on exertional rhabdomyolysis, we conducted a retrospective prevalence study with the objective of identifying patterns, circumstances, and outcomes of exertional rhabdomyolysis presentations to the emergency department (ED) during the prior 12 years. Further objectives were to determine whether the presenting patterns of patients with exertional rhabdomyolysis have changed; to assess for any trends indicating that particular activities initiate exertional rhabdomyolysis; to gather demographic information on exertional rhabdomyolysis admittances for sex and age; and to gather information regarding admittance, ED processing, and discharge of patients with exertional rhabdomyolysis. We hypothesized that presentations of exertional rhabdomyolysis would reflect the increasing popularity of extreme exercise regimens seen in the wider community.

\section{METHODS}

This study was a retrospective medical records review of presentations to the Princess Alexandra Hospital ED, a tertiary-level, adult-only ED located in Brisbane, Australia, with 61,000 primary presentations annually. Since 2005 , presentations to the ED have been documented using the electronic databases Emergency Department Information System and FirstNet (Cerner Inc.). The laboratory data were extracted from the AUSLAB laboratory information system. Ethical approval for this study was received from the Human Research Ethics Committees at both Metro South Health (HREC/16/ QPAH/310) and the University of Queensland (approval 2016-SOMILRE-0186).

Patients were included in the analysis if they had a positive urinary myoglobin or a CK level $>1,000 \mathrm{U} / \mathrm{L}$ within 2 days of their presentation or hospital admittance, were $>17$ years old, and their medical records referenced physical exertion as an inciting cause of their presentation. Case notes were reviewed to identify the underlying cause of the rhabdomyolysis, and only cases caused by exertion were included in the study.

Exclusion criteria included any presentation of rhabdomyolysis that could be attributed to another cause such as myocardial infarction, pneumonia, trauma, stroke, overdose, seizure, and electrocution. Also excluded were patients for whom the inciting problem was initially attributed to exertion but was subsequently deemed to be related to cardiac conditions, drug overdose, or stroke.

All selected patients were classified into 7 subcategories relating to the etiology of their exertional rhabdomyolysis: gym, marathon/running, manual labor, military/police training, team sports, walking/heat, and other. Demographic information and hospital admission and discharge data were collected. Variables were analyzed using appropriate descriptive statistics of frequencies, percentages, and means with standard deviations.

\section{RESULTS}

A total of 89 patients were identified as having exertional rhabdomyolysis between March 2005 and December 2016 (Figure 1). An increasing number of total ED presentations, number of rhabdomyolysis presentations, and number of exertional rhabdomyolysis presentations occurred during this 12-year period. The increase in exertional rhabdomyolysis presentations is reflected in the percentage of exertional rhabdomyolysis ED presentations compared to total ED presentations during the study period (Figure 2). The number of exertional rhabdomyolysis presentations increased from a low of 0.28 per 10,000 ED presentations in 2005 to a peak of 3.5 per 10,000 in 2015 . Of the 89 total exertional rhabdomyolysis presentations, $53.9 \%$ were attributable to gym-based workouts comprised of activities such as weight lifting, personal trainer-guided exercise, and high-intensity workouts such as CrossFit.

As shown in Table 1, $>50 \%$ of patients presented on Thursday, Friday, and Saturday and were most likely to arrive during the daytime hours (0701-1600) as walk-in patients. Half of the patients who arrived were either discharged directly from the ED or sent to short stay. Three of the 21 patients sent to short stay were subsequently admitted. The medical record identified the time of the inciting event in 73 of the 89 cases (82.0\%). Of the patients with an inciting time identified, less than half $(42.5 \%)$ presented on the same day or the day after the inciting event (Table 2). The remainder presented later, with $16.4 \%$ presenting more than a week after the inciting event.

\section{DISCUSSION}

The increasing rate of exertional rhabdomyolysis presentations to the Princess Alexandra Hospital ED from 2005-2016, in addition to confirming the study's hypothesis, adds to the little information available in the literature regarding instances of exertional rhabdomyolysis. The 4-fold increase from 2011 2014 reported in one of the Norwegian studies ${ }^{11}$ is more than the 3-fold increase during the same period at our site. Reductions in presentations compared to prior years were seen in 2010 and 2016. The significance of these decreases is not clear and would require longitudinal analysis to determine if they were attributable to natural yearly variation or represented a change in presenting patterns. Notwithstanding the fluctuation, the overall trend for increase is unequivocal.

In this study, both CK and urinary myoglobin concentrations were highly variable. This variability is likely a reflection of individual physiologic differences, the large variation in sampling time relative to the inciting incident, and discrepancies in the quantities of underlying muscle damage.

A 2016 metaanalysis indicated that patients with severe rhabdomyolysis (defined as $\mathrm{CK}>15,000 \mathrm{U} / \mathrm{L}$ ) were at the greatest risk of kidney damage, with a receiver operating characteristic curve for CK predicting an acute kidney injury occurrence of 0.75 (95\% confidence interval $0.71-0.79){ }^{14}$ The predictive value was even higher in cases relating to crush injuries, suggesting variability in subcategories of rhabdomyolysis. A follow-up study regarding progression to acute renal failure may be worthwhile, considering that the average CK of patients at Princess Alexandra Hospital was higher than the predictive value of $15,000 \mathrm{U} / \mathrm{L}$ identified in the literature. ${ }^{14}$ 


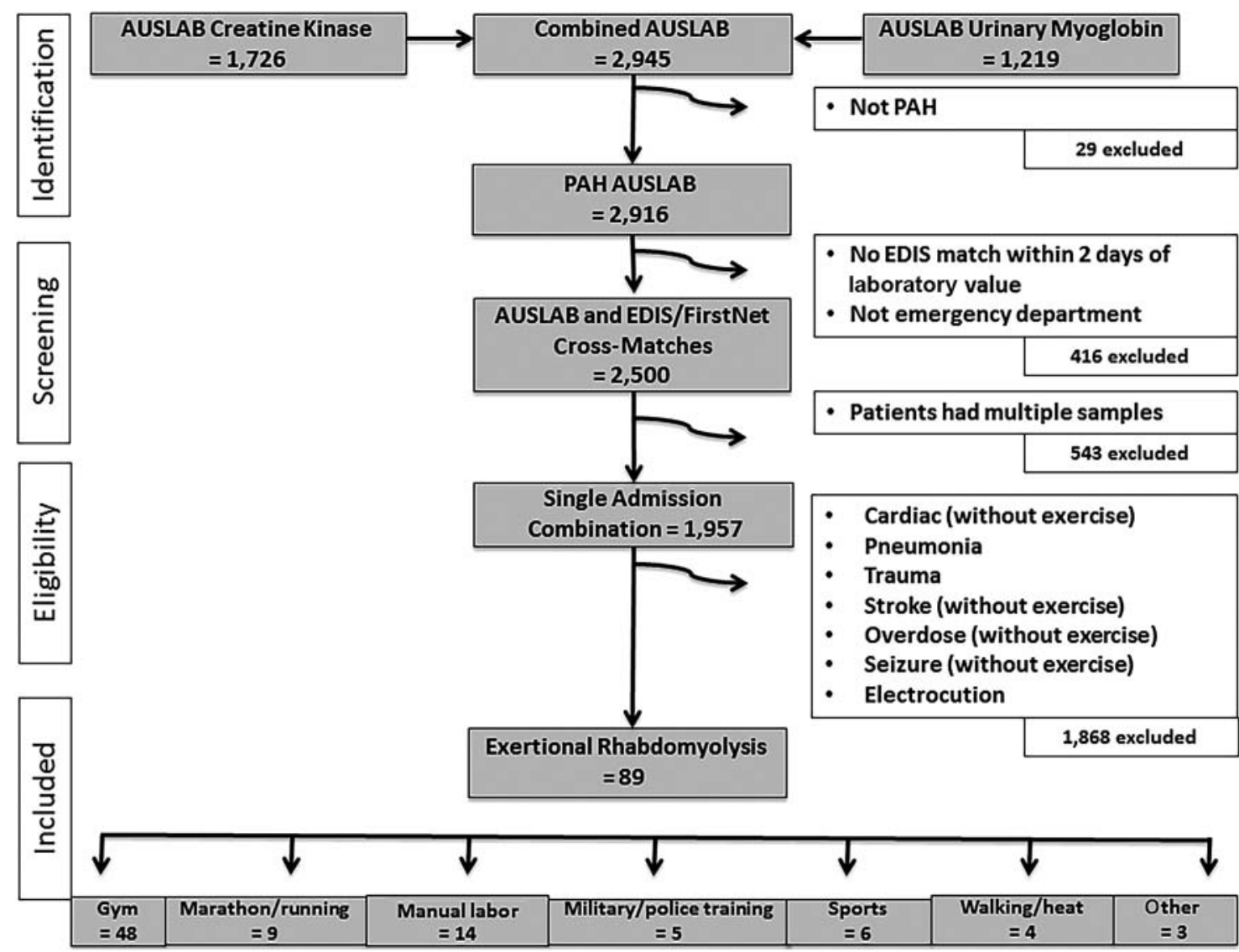

Figure 1. Data recorded in the Emergency Department Information System (EDIS/FirstNet) and AUSLAB laboratory databases at the Princess Alexandra Hospital (PAH) emergency department were used to identify, screen, determine eligibility, and categorize exertional rhabdomyolysis to identify 89 presentations with 7 major inciting subcategories.

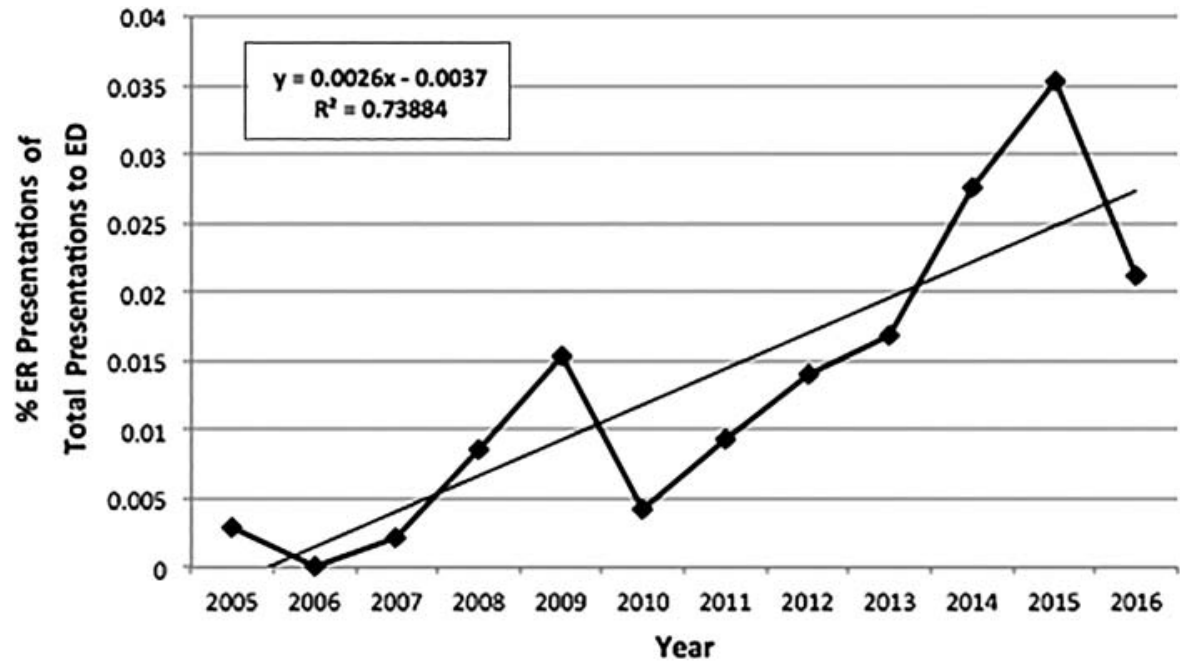

Figure 2. The percentage of exertional rhabdomyolysis (ER) cases of total emergency department (ED) presentations to Princess Alexandra Hospital is shown for the period 2005-2016. The straight line represents the linear trend for the duration of the study. 
Table 1. Demographic, Presenting, and Discharge Patterns of Patients With Exertional Rhabdomyolysis at Princess Alexandra Hospital, March 2005 to December 2016

\begin{tabular}{|c|c|}
\hline Variable & $\begin{array}{c}\text { Number of } \\
\text { Patients } n=\mathbf{8 9} \\
\end{array}$ \\
\hline \multicolumn{2}{|l|}{ Age, years } \\
\hline$<40$ & $70(78.7)$ \\
\hline $40-60$ & $17(19.1)$ \\
\hline$>60$ & $2(2.3)$ \\
\hline Sex, male & $63(70.8)$ \\
\hline \multicolumn{2}{|l|}{ Day of presentation } \\
\hline Sunday & $6(6.7)$ \\
\hline Monday & $7(7.9)$ \\
\hline Tuesday & $15(16.9)$ \\
\hline Wednesday & $11(12.4)$ \\
\hline Thursday & $17(19.1)$ \\
\hline Friday & $16(18.0)$ \\
\hline Saturday & $17(19.1)$ \\
\hline \multicolumn{2}{|l|}{ Mode of arrival } \\
\hline Walk-in & $66(74.1)$ \\
\hline Ambulance & $20(22.5)$ \\
\hline Police & $3(3.4)$ \\
\hline \multicolumn{2}{|l|}{ Time of arrival } \\
\hline $0701-1600$ & $45(50.6)$ \\
\hline $1601-2300$ & $35(39.3)$ \\
\hline $2301-0700$ & $9(10.1)$ \\
\hline $\begin{array}{l}\text { Mean time in the emergency } \\
\text { department, minutes } \pm S D\end{array}$ & $363 \pm 294.2$ \\
\hline \multicolumn{2}{|l|}{ Discharge destination } \\
\hline Admitted & $42(47.2)$ \\
\hline Discharged & $23(25.8)$ \\
\hline Short stay & $21(23.6)$ \\
\hline Transfer to another hospital & $2(2.3)$ \\
\hline Left without discharge & $1(1.1)$ \\
\hline \multicolumn{2}{|c|}{ Destination of ambulance arrivals $(n=20)$} \\
\hline Not admitted & $13(65.0)$ \\
\hline Admitted & $7(35.0)$ \\
\hline \multicolumn{2}{|c|}{ Destination of short-stay patients $(n=21)$} \\
\hline Not admitted & $18(85.7)$ \\
\hline Admitted & $3(14.3)$ \\
\hline \multicolumn{2}{|c|}{ Length of stay if admitted, days $(n=42)$} \\
\hline 1 & $16(38.1)$ \\
\hline 2 & $8(19.1)$ \\
\hline 3 & $8(19.1)$ \\
\hline 4 & $6(14.3)$ \\
\hline 5 & $2(4.8)$ \\
\hline 6 & 0 \\
\hline 7 & $1(2.4)$ \\
\hline 8 & $1(2.4)$ \\
\hline
\end{tabular}

Data are presented as $\mathrm{n}(\%)$ unless otherwise noted.
In regard to urinary myoglobin, $38.5 \%$ of the urinary myoglobin results did not indicate exertional rhabdomyolysis where $\mathrm{CK}$ results were $>1,000 \mathrm{U} / \mathrm{L}$. The literature is not consistent regarding the ability of urinary myoglobin to predict progression to acute renal failure. A 2009 literature review found that although the sensitivity for urinary myoglobin in most of the 8 studies they selected was $100 \%$, the number of false-positive results was equal to or greater than the true-positive results in all but one of the studies. ${ }^{15}$ The identification of predictive values for acute renal failure in cases of rhabdomyolysis can be vital to mitigating complications and initiating preventive treatments. Therefore, an evaluation of the role of these biomarkers in exertional rhabdomyolysis would be a valuable investigation in future studies.

Our exertional rhabdomyolysis cohort was younger than the general ED population, with $78.7 \%$ of patients $<40$ years old compared to $44.1 \%$ in the general ED population (data on file). This age range aligns with research on exertional rhabdomyolysis published in 1994 that identified 35 male patients in a retrospective medical records study with an average age of $24.4 \pm 5.4$ years. ${ }^{16}$ Similarly, the male predominance in our exertional rhabdomyolysis cohort also aligns with the literature. ${ }^{17}$ This unequal sex distribution may be partly attributable to the greater number of males vs females participating in predisposing activities. One study found a male predominance $(68.9 \%)$ in participants across 5 sports in the Australian state of Victoria. ${ }^{18}$ Even in highintensity activities such as CrossFit, in which the sex divide is not as exaggerated, males have been shown to be more likely to sustain an injury and less likely to ask for guidance from personal trainers than females. ${ }^{19}$ Another argument for the male predominance in exertional rhabdomyolysis is differences in the distribution of skeletal muscle, fat, and inflammatory responses between the sexes. Animal models have shown female rats to have lower degrees of muscle damage following extreme exertion. ${ }^{17}$ In particular, the decreased inflammatory responses to muscle damage in females have been attributed to estrogen because of its structural similarities to vitamin $E$ that may enable it to exhibit similar capabilities in suppressing oxidative stress. ${ }^{20}$ However, some contradictory research has indicated greater muscle damage in women performing eccentric exercises rather than less damage that would be predicted from the attributed action of estrogen. ${ }^{21}$

In contrast with the larger cohort of all 89 patients with exertional rhabdomyolysis $(70.8 \%$ male), the ratio of male to female presentations for the gym-induced exertional rhabdomyolysis subcategory $(n=48)$ was almost equal $(52.1 \%$ male vs $47.9 \%$ female). This finding may suggest that women may have a similar biophysical predisposition to men influencing susceptibility in the gym environment, but patterns seen in the manual labor category are accounted for by the male predominance in this profession. $^{22}$

Although several types of exercise were associated with the exertional rhabdomyolysis presentations in our cohort, gym workouts were the inciting cause for more than half of the presentations. This finding is in alignment with our study hypothesis that the increasing prevalence of exertional rhabdomyolysis potentially stems from the heightened popularity and the 24/7 availability of gyms and high-intensity workouts by individuals who perhaps have minimal scientific 
Table 2. Selected Comparisons of All Patients With Exertional Rhabdomyolysis (ER) to Patients With Gym-Based ER and Manual Labor ER

\begin{tabular}{|c|c|c|c|}
\hline Variable & All ER & Gym-Based ER & Manual Labor ER \\
\hline \multicolumn{4}{|c|}{ Time between inciting event and presentation, days ${ }^{\mathrm{a}}$} \\
\hline Same day & $22(30.1)$ & $8(17.8)$ & $7(70.0)$ \\
\hline 1 & $9(12.3)$ & $4(8.9)$ & $2(20.0)$ \\
\hline 2 & $9(12.3)$ & $7(15.6)$ & 0 \\
\hline 3 & $7(9.6)$ & $5(11.1)$ & $1(10.0)$ \\
\hline 4 & $6(8.2)$ & $6(13.3)$ & 0 \\
\hline 5 & $4(5.5)$ & $4(8.9)$ & 0 \\
\hline 6 & $4(5.5)$ & $2(4.4)$ & 0 \\
\hline 7 & $7(9.6)$ & $6(13.3)$ & 0 \\
\hline 8 & $2(2.7)$ & $2(4.4)$ & 0 \\
\hline 2 weeks+ & $3(4.1)$ & $1(2.2)$ & 0 \\
\hline Sex, male & $63(70.8)$ & $25(52.1)$ & $14(100.0)$ \\
\hline \multicolumn{4}{|l|}{ Time of arrival } \\
\hline 0701-1600 & $45(50.6)$ & $27(56.3)$ & $5(35.7)$ \\
\hline $1601-2300$ & 35 (39.3) & $16(33.3)$ & $7(50.0)$ \\
\hline 2301-0700 & $9(10.1)$ & $5(10.4)$ & $2(14.3)$ \\
\hline Mean creatine kinase, $U / L \pm S D^{b}$ & $28,061 \pm 47,912.7$ & $29,732 \pm 34,813.2$ & $17,369 \pm 31,968.0$ \\
\hline \multicolumn{4}{|l|}{ Urinary myoglobin, mg/ $\mathrm{L}^{\mathrm{c}}$} \\
\hline$<5$, negative & $20(38.5)$ & 7 (26.9) & $4(40.0)$ \\
\hline$>5$, positive & $32(61.5)$ & $19(73.1)$ & $6(60.0)$ \\
\hline
\end{tabular}

Data are presented as $n(\%)$ unless otherwise noted.

aThe medical records for 16 patients did not specify when the inciting event occurred $(n=73)$.

${ }^{b}$ Creatine kinase values were not available for 15 patients $(n=74)$.

'Urinary myoglobin values were not available for 37 patients $(n=52)$.

training or support. A 2016 article commenting on the Aalborg et al paper ${ }^{11}$ proposed that the trends observed in Norway may also be a consequence of lifestyle patterns composed of sedentary daily lives and widespread engagement in organized fitness that often emphasizes weight training, large numbers of repetitions, and eccentric movements. ${ }^{12}$ Support for this shift in exercise styles comes from a worldwide survey of fitness trends showing that high-intensity interval training was the most popular exercise type in 2014 and body weight training was the most popular in $2015 .{ }^{23}$ Participants in gym workouts possibly represent a wider range of fitness levels than participants in other sports for which a certain level of skill and fitness is required. Consequently, gym cohorts likely include individuals who are inexperienced and have a lower level of physical strength, endurance, and athletic training than participants in most sports. ${ }^{24}$

In addition to gym-induced exertional rhabdomyolysis, the literature reports incidences of exertional rhabdomyolysis related to running, police and military training, and team sports. However, the second largest subcategory in our study is manual labor-induced exertional rhabdomyolysis for which we found no literature. The exertional rhabdomyolysis cohort from Princess Alexandra Hospital included 14 individuals who presented with classic signs of rhabdomyolysis following physical exertion and exposure to heat on the work- site. Exertional rhabdomyolysis has often been referred to as "white-collar rhabdomyolysis" as it was considered to primarily impact educated individuals who could fit workouts into their schedule and who would have the energy to engage in high-intensity exercise at the end of a workday. ${ }^{25}$ However, our study shows that extreme physical exertion on the job can induce exertional rhabdomyolysis, particularly in locations such as Queensland where high temperatures combined with high humidity are common.

A number of patients presented to the ED several days after the inciting event, indicating that the physical impact and the diagnosable levels of CK and urinary myoglobin can persist for a substantial amount of time in exertional rhabdomyolysis. This finding is supported by research that monitored serum CK and serum myoglobin excretion in 13 patients with rhabdomyolysis and showed CK persisting at $66 \%$ of the first-day value after 24 hours and at $46 \%$ after 48 hours and myoglobin at $35 \%$ and $22 \%$, respectively. ${ }^{26}$ A related question is how many individuals with symptoms and diagnostically elevated laboratory values choose to manage their exertional rhabdomyolysis at home and what implications this self-care has on kidney function. Future studies may be able to evaluate these possibilities by taking blood and urine samples and administering surveys at gyms to identify cases of exertional rhabdomyolysis in patients who do not present to the ED. 
Approximately half $(47.2 \%)$ of the patients in this study who presented with exertional rhabdomyolysis were admitted to Princess Alexandra Hospital. Their average length of stay was $2.51 \pm 1.70$ days ( $n=42$, range $1-8$ days), and $42.9 \%$ stayed for 3 or more days, suggesting that at least half of the individuals experienced symptoms warranting longer-term surveillance.

We only investigated admission data and patient trends; assumptions about the severity of cases can only be made based on whether patients were admitted to the hospital and their length of stay. Future analyses of exertional rhabdomyolysis severity could examine progression to acute renal failure, commonly considered to be the most serious complication of rhabdomyolysis. Ward and Kahn, respectively, reported that $33 \%$ and $16.5 \%$ of patients with rhabdomyolysis developed acute renal failure. ${ }^{27,28}$ Long-term survival rates have been estimated as $60 \%-80 \%$ following diagnosis of acute renal failure. ${ }^{27-29}$

To our knowledge, this study is the largest analysis of exertional rhabdomyolysis presentations to the ED. However, the analysis has several limitations because of the nature of retrospective medical record studies. For instance, only 73 medical records contained sufficient information to determine the time between the inciting incident and presentation to the ED, and not all of the records consistently listed substances (medications, alcohol, supplements) that may have predisposed individuals to develop exertional rhabdomyolysis. Therefore, large multicenter studies that include a prospective assessment of factors that influence the development of exertional rhabdomyolysis should be conducted.

\section{CONCLUSION}

This study provides evidence that the number of presentations of exertional rhabdomyolysis to the ED in the Brisbane, Australia area is increasing, as well as information regarding etiology, presentation, and ED processing of admissions. While some of the data, such as age ranges of patients, can be found in the literature, other demographic information-such as the sex distribution in our gym-induced exertional rhabdomyolysis cohort-challenges the currently held belief that exertional rhabdomyolysis is a maledominated condition.

We conclude that the popularity of high-intensity workouts accounts for the increasing number of exertional rhabdomyolysis presentations; however, a variety of other potentially contributing factors ought to be investigated. The increasing frequency of exertional rhabdomyolysis is not well documented and warrants further investigation. Identification of what workout fads may be related to the increase in exertional rhabdomyolysis is important to prevention and early identification that can be vital in avoiding complications such as acute renal failure.

\section{ACKNOWLEDGMENTS}

Ms Knafl would like to thank the University of Queensland Advantage Office for its assistance with this project. $\mathrm{Mr}$ Hughes is currently supported by an Emergency Medicine Foundation Capacity Building Grant (EMCB-402R23-2015) under which he is employed. Otherwise, the authors have no financial or proprietary interest in the subject matter of this article.

\section{REFERENCES}

1. Giannoglou GD, Chatzizisis YS, Misirli G. The syndrome of rhabdomyolysis: pathophysiology and diagnosis. Eur J Intern Med. 2007 Mar;18(2):90-100.

2. Chavez LO, Leon M, Einav S, Varon J. Beyond muscle destruction: a systematic review of rhabdomyolysis for clinical practice. Crit Care. 2016 Jun 15;20(1):135. doi: 10.1186/s13054016-1314-5.

3. Chatzizisis YS, Misirli G, Hatzitolios Al, Giannoglou GD. The syndrome of rhabdomyolysis: complications and treatment. Eur J Intern Med. 2008 Dec;19(8):568-574. doi: 10.1016/j.ejim.2007.06.037.

4. Bagley $\mathrm{WH}$, Yang $\mathrm{H}$, Shah $\mathrm{KH}$. Rhabdomyolysis. Intern Emerg Med. 2007 Oct;2(3):210-218.

5. Cho J. Acute exertional rhabdomyolysis. J Orthop Sports Phys Ther. 2013 Dec;43(12):932. doi: 10.2519/jospt.2013.0420.

6. Update: exertional rhabdomyolysis, active component, U.S. Armed Forces, 2010-2014. MSMR. 2015 Mar;22(3):22-25.

7. Bruso JR, Hoffman MD, Rogers IR, Lee L, Towle G, Hew-Butler T. Rhabdomyolysis and hyponatremia: a cluster of five cases at the 161-km 2009 Western States Endurance Run. Wilderness Environ Med. 2010 Dec;21(4):303-308. doi: 10.1016/j. wem.2010.06.012.

8. Chen CY, Lin YR, Zhao LL, et al. Clinical spectrum of rhabdomyolysis presented to pediatric emergency department. BMC Pediatr. 2013 Sep 3;13:134. doi: 10.1186/1471-2431-13-134.

9. Ramme AJ, Vira S, Alaia MJ, Van De Leuv J, Rothberg RC. Exertional rhabdomyolysis after spinning: case series and review of the literature. J Sports Med Phys Fitness. 2016 Jun;56 (6):789-793.

10. Dekeyser B, Schwagten V, Beaucourt L. Severe rhabdomyolysis after recreational training. Emerg Med J. 2009 May;26(5):382383. doi: 10.1136/emj.2008.065771.

11. Aalborg C, Rød-Larsen C, Leiro I, Aasebø W. An increase in the number of admitted patients with exercise-induced rhabdomyolysis [in Norwegian]. Tidsskr Nor Laegeforen. 2016 Oct 11;136(18):1532-1536.

12. Fardal H, Gøransson LG. Exercise-induced rhabdomyolysis - a new trend [in Norwegian]? Tidsskr Nor Laegeforen. 2016 Oct 11;136(18):1537-1541.

13. Glassman G. CrossFit induced rhabdo. CrossFit J. 2005 Oct;38:13. http://library.crossfit.com/free/pdf/38_05_cf_rhabdo.pdf. Accessed June 7, 2018.

14. Safari S, Yousefifard M, Hashemi B, et al. The value of serum creatine kinase in predicting the risk of rhabdomyolysisinduced acute kidney injury: a systematic review and metaanalysis. Clin Exp Nephrol. 2016 Apr;20(2):153-161. doi: 10.1007/ s10157-015-1204-1.

15. Rodríguez-Capote K, Balion CM, Hill SA, Cleve R, Yang L, El Sharif A. Utility of urine myoglobin for the prediction of acute renal failure in patients with suspected rhabdomyolysis: a systematic review. Clin Chem. 2009 Dec;55(12):2190-2197. doi: 10.1373/clinchem.2009.128546.

16. Sinert R, Kohl L, Rainone T, Scalea T. Exercise-induced rhabdomyolysis. Ann Emerg Med. 1994 Jun;23(6):1301-1306.

17. Clarkson PM, Hubal MJ. Are women less susceptible to exercise-induced muscle damage? Curr Opin Clin Nutr Metab Care. 2001 Nov;4(6):527-531.

18. Eime RM, Harvey JT, Charity MJ, Payne WR. Population levels of sport participation: implications for sport policy. BMC Public Health. 2016 Aug 9;16:752. doi: 10.1186/s12889-016-3463-5.

19. Weisenthal BM, Beck CA, Maloney MD, DeHaven KE, Giordano $B D$. Injury rate and patterns among CrossFit athletes. Orthop J Sports Med. 2014 Apr 25;2(4):2325967114531177. doi: $10.1177 / 2325967114531177$. 
20. Tiidus PM. Can estrogens diminish exercise induced muscle damage? Can J Appl Physiol. 1995 Mar;20(1):26-38.

21. Maclntyre DL, Reid WD, Lyster DM, McKenzie DC. Different effects of strenuous eccentric exercise on the accumulation of neutrophils in muscle in women and men. Eur J Appl Physiol. 2000 Jan;81(1-2):47-53.

22. Australian Bureau of Statistics. 6291.0.55.003 - Labour Force, Australia. http://www.abs.gov.au/ausstats/abs@.nsf/mf/6291.0 .55.003. Accessed June 7, 2018.

23. Thompson WR. Worldwide survey of fitness trends for 2015: what's driving the market? ACSMs Health Fit J. 2014 Nov/Dec;18 (6):8-17.

24. Cooperman S. Getting fit, even if it kills you. The New York Times. http://www.nytimes.com/2005/12/22/fashion /thursdaystyles/getting-fit-even-if-it-kills-you.html. Published December 22, 2005. Accessed June 7, 2018.
25. Knochel JP. Catastrophic medical events with exhaustive exercise: "white collar rhabdomyolysis". Kidney Int. 1990 Oct;38 (4):709-719.

26. Lappalainen $H$, Tiula $E$, Uotila $L$, Mänttäri M. Elimination kinetics of myoglobin and creatine kinase in rhabdomyolysis: implications for follow-up. Crit Care Med. 2002 Oct;30(10):22122215.

27. Ward MM. Factors predictive of acute renal failure in rhabdomyolysis. Arch Intern Med. 1988 Jul;148(7): 1553-1557.

28. Khan FY. Rhabdomyolysis: a review of the literature. Neth J Med. 2009 Oct;67(9):272-283.

29. Bosch X, Poch E, Grau JM. Rhabdomyolysis and acute kidney injury. N Engl J Med. 2009 Jul 2;361(1):62-72. doi: 10.1056/ NEJMra0801327.

This article meets the Accreditation Council for Graduate Medical Education and the American Board of Medical Specialties Maintenance of Certification competencies for Patient Care and Medical Knowledge. 